\title{
Soziale Daseinsvorsorge und Haushaltskonsolidierung
}

\section{Wir müssen in die Zukunft investieren}

\author{
Karl Koch und Franz Segbers
}

Dr. Karl Koch leitet das Referat Sozialpolitische Grundsatzfragen im Caritasverband für die Diözese Limburg e. V.

E-Mail karl.koch@dicv-limburg.de Prof. Dr. Franz Segbers leitet das Referat Arbeit, Ethik und Sozialpolitik im Diakonischen Werk Hessen und Nassau. Er ist zudem Hochschullehrer für Sozialethik am Fachbereich Evangelische Theologie der Universität Marburg.

E-Mail Franz.Segbees@online.de
Die Finanzkrise insbesondere der Kommunen und die Schuldenbremse für den Bund und die Länder gefährden die soziale Daseinsvorsorge und eine bedarfsdeckende Bereitstellung sozialer Dienstleistungen.

Die Bundesrepublik Deutschland definiert sich laut Grundgesetz als demokratischer Rechts- und Sozialstaat (Art. 20 und 28 GG). Auch die Hessische Landesverfassung enthält wirtschaftliche und soziale Rechte, die den Gesetzgeber zu entsprechenden sozialen Aktivitäten verpflichten. Grundgesetz und Landesverfassung bestimmen die Ziele und Aufgaben staatlicher Politik. Damit der Staat seine Aufgaben erfüllen kann, benötigt er Einnahmen. Das Recht, von den Bürgern des Staates Abgaben zu erheben, ist gleichzeitig Bedingung und notwendige Folge der Existenz des Staates. Ohne »Finanzgewalt « kann kein Staat bestehen.

Zwischen den öffentlichen Aufgaben, die der Staat erfüllen soll, und den öffentlichen Ausgaben besteht ein unmittelbarer Zusammenhang. Die Höhe der Ausgaben und die Art der Ausgaben hängen direkt davon ab, welche Ziele der Staat erreichen will und welche Aufgaben der Staat übernehmen soll.

Bisher bestand Konsens darüber, dass es Aufgabe des Staates ist, die soziale Daseinsvorsorge zu übernehmen und zu gewährleisten. Soziale Daseinsvorsorge meint die Bereitstellung von Gütern und Dienstleistungen zur Grundsicherung des Lebens und zur Vorsorge gegen Risiken. Diese Güter und Dienstleistungen sind auch dann zur Verfügung zu stellen, wenn sie keinen Gewinn abwerfen und nicht marktförmig erbracht werden können. Derartige Güter und Dienstleistungen sind beispielsweise eine funktionierende Verkehrsinfrastruktur, eine funktionierende Kommunikationsstruktur (Post, Telekommunikation), ein bedarfsdeckendes Angebot an sozialen Diensten und Einrichtungen. Die Wohlfahrtsver- bände stellen eine Vielzahl an Diensten und Einrichtungen der sozialen Daseinsvorsorge zur Verfügung für die der Staat die Gewährleistungsfunktion übernimmt.

Die Wirtschafts- und Finanzkrise, die noch nicht überwunden ist, hat die Verschuldung der öffentlichen Haushalte in die Höhe getrieben, so dass die Konsolidierung der Haushalte inzwischen oberste Priorität erhalten hat. Die beabsichtigte Konsolidierungspolitik wird die soziale Daseinsvorsorge und damit die Wohlfahrtsverbände als Leistungsanbieter nicht ungeschoren davon kommen lassen.

\section{Beispiel: Die Situation des Landes Hessen}

Wir erleben zurzeit die »Verkämmerung « der Politik, es wird also nicht zuerst gefragt, welche sozialen und ökologischen Ziele und Aufgaben die Politik $\mathrm{zu}$ erfüllen hat und welche finanziellen Mittel hierfür staatlicherseits zu mobilisieren sind, sondern die sozialen und ökologischen Ziele und Aufgaben sind in der Gefahr, dem Ziel der Haushaltskonsolidierung untergeordnet zu werden. »Der auf unabsehbare Zeit hegemonial institutionalisierte Defizit- und Schuldendiskurs wird Staat, Politik und soziale Demokratie auf Dauer als Problem statt als Lösung definieren, trotz und gerade wegen der in der Weltwirtschaftskrise am Ende des neoliberalen Zeitalters von neuem erwiesenen Unentbehrlichkeit der öffentlichen Gewalt für die Funktionsfähigkeit der privatkapitalistischen Wirtschaft." (Streeck/Mertens 2010: 32)

Hessen hat 2010 bei den bereinigten Gesamtausgaben ein Haushaltssoll von 21,5 Milliarden Euro und bereinigte Gesamteinnahmen von 18,2 Milliarden Euro. Die Nettoneuverschuldung beträt 3,3 Milliarden Euro. Für Zinsen werden 1,4 Milliarden Euro ausgegeben und für den kommunalen Finanzausgleich sind 2,9 Milliarden Euro vorgesehen. Hessen 
hat mittlerweile 40 Milliarden Euro an Schulden angehäuft. Im Jahr 2000 betrugen die Schulden noch 24 Milliarden Euro, 2005 bereits 31 Milliarden Euro und 2010 waren es 39 Milliarden Euro. Die CDU-geführten Landesregierungen haben immer eine Verschuldungspolitik betrieben, obgleich Sie sich in der Öffentlichkeit als erfolgreiche Haushaltssanierer darstellen konnten.

Die Ausgabenentwicklung des hessischen Landeshaushaltes war in den zurückliegenden Jahren keineswegs außergewöhnlich hoch. Das Hessische Finanzministerium hat für die Jahre 2000 bis politik der verschiedenen Bundesregierungen hat die Kommunen hart getroffen. Hinzu kommt, dass die Kommunen Ausfälle beim kommunalen Finanzausgleich verkraften müssen. Erhielten die Kommunen 2009 noch 3,2 Milliarden Euro aus dem kommunalen Finanzausgleich; so erhalten sie aufgrund sinkender Einnahmen durch die Wirtschaftskrise 2010 nur noch 2,9 Milliarden Euro. Im Haushaltsentwurf 2011 werden den Kommunen dann noch einmal innerhalb des kommunalen Finanzausgleiches 198 Millionen Euro gestrichen und außerhalb des kommunalen Finanzausgleiches 160

\section{"Wir erleben zurzeit die >Verkämmerung " der Politik «}

2010 eine durchschnittliche Wachstumsrate der Ausgaben von lediglich 1,8 Prozent errechnet. Allerdings stiegen im gleichen Zeitraum die Einnahmen lediglich um durchschnittlich 0,5 Prozent (Hessisches Ministerium der Finanzen 2010: 13). Eicker-Wolf/Truger kommen in einer Studie zu dem Ergebnis, dass durch die Steuerreformen seit 1998 dem Land Hessen in 2010 rund zwei Milliarden Euro an Steuerausfällen entstehen. Und die hessischen Kommunen haben aufgrund der Steuerpolitik einen Ausfall von 1,2 Milliarden Euro (incl. Kommunalem Finanzausgleich) zu verkraften (vgl. EickerWolf/Truger, 2010: 36) Es ist also insbesondere die Steuersenkungspolitik der zurückliegenden Jahre, die die Verschuldung des Landes Hessen und der hessischen Kommunen in die Höhe trieb.

Folge der verfehlten Steuerpolitik ist, dass in Hessen die Staatsquote, die Personalausgabenquote und die Investitionsquote seit Mitte der neunziger Jahre nach unten weisen. Weitere Steuersenkungen würden die schon jetzt katastrophale Lage der öffentlichen Haushalte weiter verschärfen.

\section{Zur Situation der hessischen Kommunen}

Fast alle hessischen Kommunen kommen durch die Entwicklung bei den Einnahmen und Ausgaben in eine Überschuldungssituation. Die Steuersenkungs-
Millionen Euro. (Hessisches Ministerium der Finanzen 2010b: 7)

Doch nicht nur durch die finanzpolitischen Entscheidungen im Bund und im Land Hessen geraten die Kommunen unter Druck, sondern sie haben auch einen Ausgabenanstieg bei den Kosten der Unterkunft für SGB II-Empfängern oder bei den Pflichtaufgaben wie dem Kinderförderungsgesetz zu verkraften. So hat sich allein für die Landkreise in Hessen der Zuschussbedarf für die Kinder- und Jugendhilfe, die Sozialhilfe und das SGB II von 2002 bis 2007 um mehr als 22 Prozent oder um 222 Millionen Euro auf insgesamt 1,2 Milliarden Euro erhöht. (Hessischer Landkreistag: 2009)

Die Sozialausgaben entwickeln eine immer größere Sprengkraft für die kommunalen Haushalte. Fast ein Viertel der Gesamtausgaben bringen die Städte und Gemeinden für Sozialleistungen auf. Mehr als die Hälfte der Sozialausgaben - nämlich 57 Prozent - entfielen für die Eingliederungshilfe für behinderte Menschen. Ein Fünftel der Ausgaben entfiel auf die Grundsicherung im Alter und bei Erwerbsminderung. Hohe Aufwendungen erfordert auch das Hartz IV-Gesetz.

\section{Konsolidierungszwang durch die Schuldenbremse}

Im Jahre 2009 hat die schwarz-rote Bundesregierung eine Schuldenbremse in der Verfassung festgeschrieben. Danach dürfen sich die Länder ab 2020 nicht mehr neu verschulden. Diese Schuldenbremse übt einen erheblichen Konsolidierungsdruck auf den Landeshaushalt in Hessen aus. Mit dem Haushaltsentwurf 2011 und der mittelfristigen Finanzplanung wird nun der Einstieg in die Konsolidierungsphase eingeleitet.

Zur Umsetzung der Schuldenbremse will die CDU/FDP-Landesregierung die Einnahmen beträchtlich erhöhen: zwischen 2011 und 2020 auf jahresdurchschnittlich 2,9 Prozent (gegenüber einer realen Einnahmenerhöhung von jährlich 0,5 Prozent zwischen 2000 und 2010). Und sie will deutlich auf die Bremse treten, indem sie die Ausgaben auf jahresdurchschnittlich 1,1 Prozent reduzieren will (gegenüber 1,8 Prozent zwischen 2000 und 2010). (Hessisches Ministerium der Finanzen, 2010: 14) Die Einnahmesteigerung soll vor allem durch Wirtschaftswachstum erzielt werden eine sehr optimistische Vorstellung -, die Ausgabenreduzierung soll durch Effizienzsteigerung, Standardabsenkungen und Ausgabenkürzungen zustande kommen. Steuererhöhungen werden bisher ausgeschlossen.

\section{Forderungen der Kommunalen Spitzenverbände}

Der Hessische Landkreistag hat darauf hingewiesen, dass die Auseinanderentwicklung von Einnahmen und Ausgaben die Kommunen »in eine nicht mehr beherrschbare Haushaltssituation « bringt. (Hessischer Landkreistag 2009) Die Entscheidungen der Bundesregierung (Steuersenkungen) und der Landesregierung (Kürzung des Kommunalen Finanzausgleichs) sowie die Wirtschaftskrise ließen die Einnahmen sinken, während insbesondere bei den sozialen Leistungen ein Ausgabenanstieg zu verzeichnen ist.

Diese verfahrene Situation zwingt schon seit Jahren Kommunen dazu freiwillige Leistungen $\mathrm{zu}$ streichen, Investitionen zu reduzieren, Gebühren zu erhöhen, Freizeit- und Kultureinrichtungen zu schließen oder einzuschränken. Um die finanzielle Situation der kommunalen Haushalte zu stabilisieren wird deshalb von kommunaler Seite gefordert, dass keine Kürzungen beim kommunalen Finanzausgleich vorgenommen werden, dass keine Schwächung der Gewerbesteu- 
er erfolgt, dass gesetzliche Standardvorgaben ausgesetzt oder reduziert werden, dass gesetzliche Aufgaben ausgesetzt oder reduziert werden, dass der Bund sich an den Sozialausgaben stärker beteiligen soll.

\section{Fazit: Die Wohlfahrtsverbände müssen sich einmischen}

Wenn die Wohlfahrtsverbände ein Interesse an der Sicherung einer bedarfsdeckenden sozialen Infrastruktur, an Planungssicherheit und an einer sozialen Entwicklung haben, die die soziale Spaltung nicht weiter verschärft, wird sie auch ein Interesse an einer soliden und nachhaltigen Finanzierungsgrundlage der öffentlichen Haushalte haben müssen. Die Kommunen können beispielsweise den sozialen Ausgleich nur schaffen, wenn sie über die entsprechenden Finanzmittel verfügen.

Die Liga der Freien Wohlfahrtspflege in Hessen fordert daher einen handlungsund leistungsfähigen Staat, der über ausreichende Einnahmen verfügt. Hierzu sind auch Steuererhöhungen nötig, da die Konsolidierung nicht allein über Wirtschaftswachstum, Ausgaben-, Leistungsund Standardkürzungen zu erreichen ist.

Bund, Länder und Kommunen stehen nicht nur vor der Aufgabe der Haushaltskonsolidierung, sondern sie haben auch wichtige Zukunftsfragen im Hinblick auf die soziale und ökologische Entwicklung $\mathrm{zu}$ bearbeiten. So sind in den nächsten Jahren sowohl in die ökologische Modernisierung von Wirtschaft und Gesellschaft erhebliche Mittel zu investieren als auch in Bildung, Gesundheit und soziale Sicherheit.

Es ist zu befürchten, dass durch den Konsolidierungspfad, den Bund, Länder und Gemeinden beschreiten, die vor uns liegenden Notwendigkeiten unzureichend bearbeitet werden. Dies wäre ökologisch und sozial unverantwortlich. Es wäre auch wirtschaftlich kontraproduktiv, da unzureichende ökologische Investitionen die volkswirtschaftlichen Reparaturkosten deutlich ansteigen lassen - siehe beispielsweise die volkswirtschaftlichen Kosten des Klimawandels - und unzureichende Investitionen in Bildung, Gesundheit und soziale Sicherheit die sozialen Kosten in die Höhe treiben.
Die Wohlfahrtsverbände müssen wachsam sein und sich einer Politik entgegenstellen, die mit dem Mittel der Haushaltskonsolidierung durch eine Schuldenbremse die soziale Daseinsvorsorge und den Sozialstaat infrage stellt und letztlich demontieren will.

\section{Literatur}

Eicker-Wolk, Kai/Truger, Achim, 2010: Entwicklung und Perspektiven der Kommunalfinanzen in Hessen. Eine Studie im Auftrag von verdi Hessen, Frankfurt am Main.

Hessischer Landkreistag vom 6. Mai 2009.

Hessisches Ministerium der Finanzen 2010a: Die Einführung der Schuldenbremse in Hessen. Hintergründe und Argumente (September).

Hessisches Ministerium der Finanzen 2010b, Pressekonferenz vom 7. Juli 2010.

Streeck, Wolfgang/Mertens, Daniel 2010: Politik im Defizit. Austerität als fiskalpolitisches Regime, MPIfG Discussion Paper 10/5, Köln.

Truger, Achim/ Eicker-Wolf, Kai 2009: Auswirkungen der Schuldenbremse auf die hessischen Landesfinanzen, IMK Studies 6/2009.

\section{Soziale Mindest- sicherung}

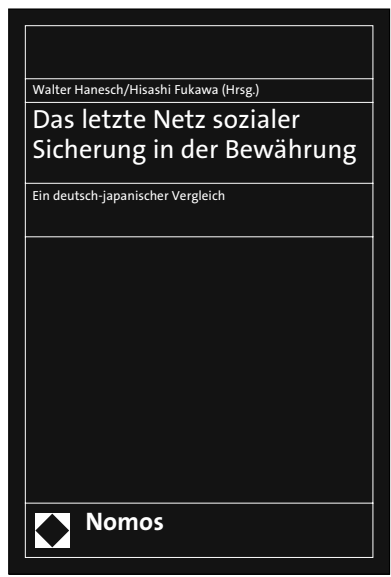

\section{Das letzte Netz sozialer Sicherung in der Bewährung} Ein deutsch-japanischer Vergleich Herausgegeben von Prof. Dr. Walter Hanesch und Prof. Dr. Hisashi Fukawa

2011, ca. 250 S., brosch., ca. 49, $-€$

ISBN 978-3-8329-5965-4

Erscheint ca. März 2011

Der vorliegende Band untersucht die letzten Netze sozialer Sicherung in Japan und Deutschland in vergleichender Perspektive. Die zentrale Aufgabe dieser sozialen Mindestsicherungssysteme liegt vor allem darin, Armut zu vermeiden und eine Wiedereingliederung in die gesellschaftliche Normalität zu ermöglichen. Mit Japan und Deutschland werden die sozialen Mindestsicherungssysteme in zwei der wirtschaftsstärksten Nationen mit sehr unterschiedlichen Sozialstaatsmodellen gegenübergestellt.

\section{Nomos}

Bitte bestellen Sie im Buchhandel oder versandkostenfrei unter $\downarrow$ www.nomos-shop.de 